\title{
Finished Pharmaceutical Product
}

National Cancer Institute

\section{Source}

National Cancer Institute. Finished Pharmaceutical Product. NCI Thesaurus. Code C42639.

A pharmaceutical product that has undergone all stages of production and testing, including packaging in its final container and labelling. 